\title{
Novel hemorrhage control technologies and their potential applicability in trauma medicine
}

\author{
Victoria Chan, Chloe Gui
}

Faculty Reviewer: Rich Hilsden, MD, MBA, CCFP, FRCSC (Department of Surgery, Division of General Surgery), Kelly Vogt, MD, MSc, FRCSC (Department of Surgery, Division of General Surgery)

\section{ABSTRACT}

Mortality due to hemorrhage is potentially preventable but remains a prevalent problem in trauma care. Despite advances in prehospital hemorrhage control, exsanguination remains the leading and second-leading cause of mortality in military and civilian trauma, respectively. Novel hemorrhage control technologies for military and civilian prehospital use include the iTClamp (iTraumaCare, Edmonton, Canada), a mechanical clamp used to close wounds; XStat (RevMedx, Wilsonville, OR), a syringe applicator that injects expandable cellulose sponges into a wound for internal compression and clotting acceleration; ResQFoam (Arsenal Medical, Watertown, MA), liquids injected into the abdominal cavity that mix and transform into an expandable solid to compress internal organ wounds; and TraumaGel (Cresilon, Brooklyn, NY), a biocompatible gel that promotes clotting and polymerizes to form a mesh to seal the wound. These 4 technologies all show promise in effective hemorrhage control, and have been designed for quick and easy use in the setting of prehospital trauma care. However, these products are still in the early stages of development with limited research data for human use. Therefore, efficiency of use, effectiveness in hemostasis, and safety should be examined for each technology to determine whether any of them warrant widespread adoption for hemorrhage control.

\section{INTRODUCTION}

Uncontrolled hemorrhage is the leading cause of military trauma deaths. ${ }^{1}$ Among civilian trauma patients, it is the second-leading cause of death ${ }^{2}$ and the leading cause of potentially preventable mortality. ${ }^{3}$ Great effort has been invested into inventing and improving hemorrhage control techniques and technologies, with new products striving towards efficient use, effective control of bleeding, and a low incidence of complications. ${ }^{1}$ In prehospital trauma care, novel technologies are often first evaluated in the military setting after evidence of effectiveness in animal studies, and if the technology proves successful, implementation into standard civilian trauma care may result. Well-established methods of trauma hemorrhage control include gauzes such as QuikClot Combat Gauze (Z-Medica, Wallingford, CT), Celox Gauze (Medtrade Products Ltd., Crewe, UK), and ChitoGauze (HemCon Medical Technologies Inc., Portland, OR), which contain substances in their mesh fibres to accelerate the clotting process. However, these products require direct manual pressure for at least 3 minutes, take time to stop the bleeding, and are not always effective. ${ }^{4}$ Therefore, techno- logical improvements are necessary to increase survival from exsanguinating traumatic wounds.

These gauze products were developed for use in the U.S. military through rigorous examinations of hemorrhage control effectiveness by the Committee on Tactical Combat Casualty Care. ${ }^{5}$ Specifically, first-generation hemostatic agents were initially approved for military use in 2003, and included QuikClot zeolite granules, which were shown to promote clotting but caused second-degree burns through its exothermic reaction. Therefore, second-generation products were subsequently developed from 2003 to 2008, such as Celox granules made of chitosan and QuikClot Combat Gauze made of kaolin, which were substances that proved to be more effective in accelerating clotting and safer than the first-generation agents. All hemorrhage control technologies developed after 2008 are considered third-generation products, and many are currently being studied to determine potential advantages over existing hemostasis techniques. The constant review of current products for improved efficacy and safety highlights the importance of the development of new technologies to control hemorrhage in military and civilian trauma care.

\section{NOVEL TECHNOLOGIES}

There are many hemostatic technologies at various stages of development; for this review, we have chosen to discuss 4 products that vary in their materials and methods of application. Each of these technologies represents a novel approach to hemorrhage control and has recently received or is pursuing U.S. Food and Drug Administration (FDA) approval.

\section{iTClamp}

The iTClamp, developed by the Canadian-based emergency medicine technology company iTraumaCare, provides a mechanical means of hemorrhage control. The device consists of two $7 \mathrm{~cm}$ bars connected by a hinge, which, when placed on a bleeding wound, act as a clamp by exerting pressure to approximate the skin and close the wound. ${ }^{6}$ Each bar contains a row of $4 \mathrm{~mm}$-long 21-gauge needles so that the device can create an air- and fluid-tight seal and remain in place once applied. ${ }^{7}$ Despite the needles, human volunteers rated the pain upon application as a 2 or 3 on a 10-point scale and reported feeling only pressure after the device was completely secured. ${ }^{7}$ The design of the iTClamp allows pressure equalization between the wound pocket and the bleeding source, and the resulting hematoma applies further pressure onto the bleeding vessels to stop the flow. ${ }^{6}$ While animal studies demonstrated no further injury by the device when left in place for several hours, ${ }^{8}$ the iTClamp is meant to 
be removed so that surgical repair can be performed on the wound. It was approved for use by Health Canada in $2012,{ }^{6}$ as well as by the FDA for use on extremities, the axilla, and the inguinal region in May 2013, ${ }^{9}$ the scalp in October $2013,{ }^{10}$ and the neck in October $2014 .{ }^{11}$

\section{XStat}

The XStat device, from the Oregon-based trauma technology company RevMedx, was created to control bleeding from junctional wounds, which are the body regions where an extremity meets with the trunk. Limb tourniquets do not adequately control hemorrhage in these regions. ${ }^{4}$ XStat is comprised of a syringe applicator filled with compact sponges that, when injected into the wound, absorb the blood and expand to fill the wound cavity within $20 \mathrm{sec}-$ onds. ${ }^{12}$ The sponges are composed of cellulose, with a radiopaque marking on one side to facilitate removal, and an outer coating of chitosan, a polysaccharide that promotes platelet aggregation and adhesion. ${ }^{4}$ Therefore, the sponges not only expand extensively to provide wound compression, removing the need for external manual pressure, but also accelerate coagulation. However, the sponges need to be removed within 4 hours to prevent limb ischemia. ${ }^{13}$ One animal study compared XStat to Combat Gauze application during severe bleeding, and described faster application time and less blood loss. ${ }^{4}$ XStat was cleared by the FDA for military use in April 2014 as well as for civilian first-responder use in December $2015,{ }^{13}$ and will be available in Canada in the near future. ${ }^{14}$

\section{ResQFoam}

While XStat is used to treat external wounds, ResQFoam was designed by Massachusetts's Arsenal Medical to stop intraabdominal hemorrhage. ${ }^{15}$ The ResQFoam device is a pistol-like appliance that contains 2 liquids in separate chambers, one in a polyol phase and the other in an isocyanate phase. ${ }^{15,16}$ When the trigger is pressed, the liquids are forced to a front chamber in the device to mix and are injected into the abdomen through the umbilicus. ${ }^{16}$ The material then transforms into a solid polyurethane phase and rapidly expands within 1 minute to conform to the intraabdominal contents and compress wound sites. ${ }^{15}$ Since surgical removal of the material is required, the foam has been designed to be nonadherent to the internal tissues and to be removed in one piece. ${ }^{15}$ Currently, only studies using this technology on swine have been published; however, a phase I clinical trial on subjects presenting with emergent intraabdominal hemorrhage was started in August 2016 and is ongoing. ${ }^{17}$ In the swine studies, a major complication observed was focal ecchymotic bowel lesions from necrosis due to the pressure exerted by the foam, which would require surgical repair during removal of the foam. ${ }^{15}$

\section{TraumaGel}

TraumaGel, invented by Joe Landolina when he was 17 years old, is a gel that can be applied to the surface of a wound to stop bleeding without external pressure. ${ }^{18}$ The gel solution contains algae cell wall polymers that form a mesh to seal the wound, as well as other constituents that promote clotting. The polymers also arrange in a way that allows the gel to take on properties of the extracellular matrix of tissues with which it comes into contact, such as the skin or the liver. ${ }^{19}$ Additionally, the gel is biocompatible and thus can be left to reabsorb. The gel was approved for veterinarian use in the summer of 2015 under the trade name VetiGel, and approval of TraumaGel for human use is currently being pursued. ${ }^{18}$

\section{CRITIQUE AND FUTURE DIRECTIONS}

Each of the 4 technologies shows promise in its applicability to trauma medicine, as each has undergone studies to demonstrate its effectiveness in hemostasis. In particular, these products are targeted for prehospital use by first responders such as paramedics, rather than in-hospital medical or surgical personnel who instead have well-established, effective techniques for definitive hemorrhage treatment. These devices were designed to be compact, simple, and quick to use, which is necessary for a prehospital hemorrhage control strategy. However, shelf life and refrigeration have not been reported. Therefore, developers must ensure that the products are able to withstand austere prehospital conditions.

TraumaGel may offer an advantage because of its simplicity of use; it only requires squeezing gel from a syringe, while iTClamp, XStat, and particularly ResQFoam would likely require significant additional training. However, the ability of TraumaGel, as a topical agent with no compressive capability, to control significant hemorrhage remains to be seen. Contrarily, while ResQFoam is able to provide substantial compression, it elicits certain concerns regarding efficacy and safety due to its invasive application and removal. Firstly, considerable education would need to be provided to first responders for safe application into the abdominal cavity. Also, its removal requires a full laparotomy, when nonoperative management of abdominal trauma is frequently possible, ${ }^{20}$ and surgery itself poses potential risks. Furthermore, in addition to the complication of bowel lesions caused by the expanding foam, other adverse effects such as respiratory compromise or decreased venous return could result from the compression. On the other hand, XStat was designed to provide compression to junctional wounds, which remains a difficult clinical problem for both first responders and trauma specialists. Therefore, while all 4 technologies demonstrate potential for future implementation into trauma care, only experience with these technologies will establish their efficacy in trauma. Often this experience is first established empirically through military usage with subsequent approval for civilian first-responder care. ${ }^{21}$

\section{CONCLUSION}

Hemorrhage control is an integral part of trauma medicine, and current research is focused on technologies that rapidly stop bleeding in order to improve survival rates. The iTClamp, XStat, ResQFoam, and TraumaGel are all novel technologies that show potential for improving prehospital hemorrhage care, as early research has demonstrated fast application, effective cessation of bleeding, and low complication rates. Because these technologies are still under development, ultimate adoption will depend on experience 
that provides data on important real-world factors such as: ease of use, effectiveness of hemostasis, impact on mortality, and complications. Nevertheless, the continual development of improved hemostasis technologies that decrease hemorrhage mortality appears promising, with the iTClamp, XStat, ResQFoam, and TraumaGel potentially having a role in the prehospital management of traumatic hemorrhage.

\section{REFERENCES}

1. Alam HB, Burris D, DaCorta JA, et al. Hemorrhage control in the battlefield: role of new hemostatic agents. Mil Med. 2005 Jan;170(1):63-9.

2. Kauvar DS, Lefering R, Wade CE. Impact of hemorrhage on trauma outcome: an overview of epidemiology, clinical presentations, and therapeutic considerations. J Trauma. 2006 Jun;60(6 Suppl):S3-11.

3. Rossaint R, Bouillon B, Cerny V, et al. Management of bleeding following major trauma: an updated European guideline. Crit Care. 2010 Apr;14(2):R52.

4. Sims K, Bowling F, Montgomery H, et al. Management of external hemorrhage in tactical combat casualty care: the adjunctive use of XStatTM compressed hemostatic sponges. J Spec Oper Med. 2016 Spring;16(1):19-28.

5. Bennett B, Littlejohn LF. Review of new topical hemostatic dressings for combat casualty care. Mil Med. 2014 May;179(5):497-514.

6. iTraumaCare. iTClamp. [Internet]. San Antonio(TX):Innovative Trauma Care;2015-2016 [cited 2016 Nov 21]. Available from: http://www. innovativetraumacare.com/itclamp.

7. Holley J. How the iTClamp works. J Emerg Med Serv. 2013 Dec;38(12):S18-23.

8. Filips D, Logsetty S, Tan J, et al. The iTClamp controls junctional bleeding in a lethal swine exsanguination model. Prehosp Emerg Care. 2013 Oct-Dec;17(4):562-32.

9. U. S. Food and Drug Administration. 510(k) Premarket Notification, Innovative Trauma Care, Inc., iTClampTM50. [Internet]. Silver Spring(MD):U. S. Food and Drug Administration;2013 May 14 [cited 2016 Nov 21]. Available from: https://www.accessdata.fda.gov/cdrh docs/pdf12/K123551.pdf.

10. U. S. Food and Drug Administration. 510(k) Premarket Notification, Innovative Trauma Care, Inc., iTClampTM50. [Internet]. Silver Spring(MD):U. S. Food and Drug Administration;2013 Oct 28 [cited 2016 Nov 21]. Available from: https://www.accessdata.fda.gov/cdrh docs/pdf13/K132651.pdf.

11. U. S. Food and Drug Administration. 510(k) Premarket Notification, Innovative Trauma Care, Inc., iTClampTM50. [Internet]. Silver Spring(MD):U. S. Food and Drug Administration;2014 Oct 9 [cited 2016 Nov 21]. Available from: https://www.accessdata.fda.gov/cdrh_docs/ pdf14/K140805.pdf.

12. RevMedx. XStat ${ }^{\circledast}$. [Internet]. Wilsonville(OR):RevMedx;2016 [cited 2016 Nov 21]. Available from: http://www.revmedx.com/xstat-dressing.

13. U. S. Food and Drug Administration. De novo classification request for XStat. [Internet]. Silver Spring(MD):U. S. Food and Drug Administration;2013 Jan 28 [cited 2016 Nov 21]. Available from: http://www. accessdata.fda.gov/cdrh_docs/reviews/K130218.pdf.

14. Toronto Star Touch. X marks the shot. [Internet]. Toronto(ON):Toronto Star Newspapers Ltd.;2015 Dec 15 [cited 2016 Nov 23]. Available from: http://startouch.thestar.com/screens/19f63afo-4597-4040-a397ef07726126bl\%7C_0.html.
15. Chang JC, Holloway BC, Zamisch M, et al. ResQFoam for the treatment of non-compressible hemorrhage on the front line. Mil Med. 2015 Sep;180(9):932-3.

16. Arsenal Medical. Arsenal trauma foam system. [Internet]. Watertown(MA):Arsenal Medical;2014 Sep 3 [cited 2016 Nov 21]. Available from: http://www.fda.gov/downloads/MedicalDevices/NewsEvents/ WorkshopsConferences/UCM415150.pdf.

17. ClinicalTrials.gov. REVIVE: Reducing exsanguination via in-vivo expandable foam (REVIVE). [Internet]. Decatur(GA):ClinicalTrials. gov;2016 [cited 2016 Nov 21]. Available from: https://clinicaltrials.gov/ ct2/show/NCT02880163.

18. Cresilon. VetiGel ${ }^{\mathrm{TM}}$ Hemostatic Gel Kit. [Internet]. Brooklyn(NY):Cresilon, Inc.;2016 [cited 2016 Nov 21]. Available from: https://cresilon. com/index.php/vetigel/.

19. Landolina JA. In-situ cross-linkable polymeric compositions and methods thereof. Pine Bush(NY);US 20140287061 Al, 2014 Sep 25.

20. Chaudery M, Clark J, Wilson MH, et al. Traumatic intra-abdominal hemorrhage control: Has current technology tipped the balance toward a role for prehospital intervention? J Trauma Acute Care Surg. 2015 Jan;78(1):153-63.

21. Halevi G. Military medicine and its impact on civilian life. Res Trends [Internet]. 2013 Sep [cited 2016 Dec 20];(34):[about 17 screens]. Available from: https://www.researchtrends.com/issue-34-september-2013/ military-medicine-and-its-impact-on-civilian-life/.

\section{MEDICALMART}

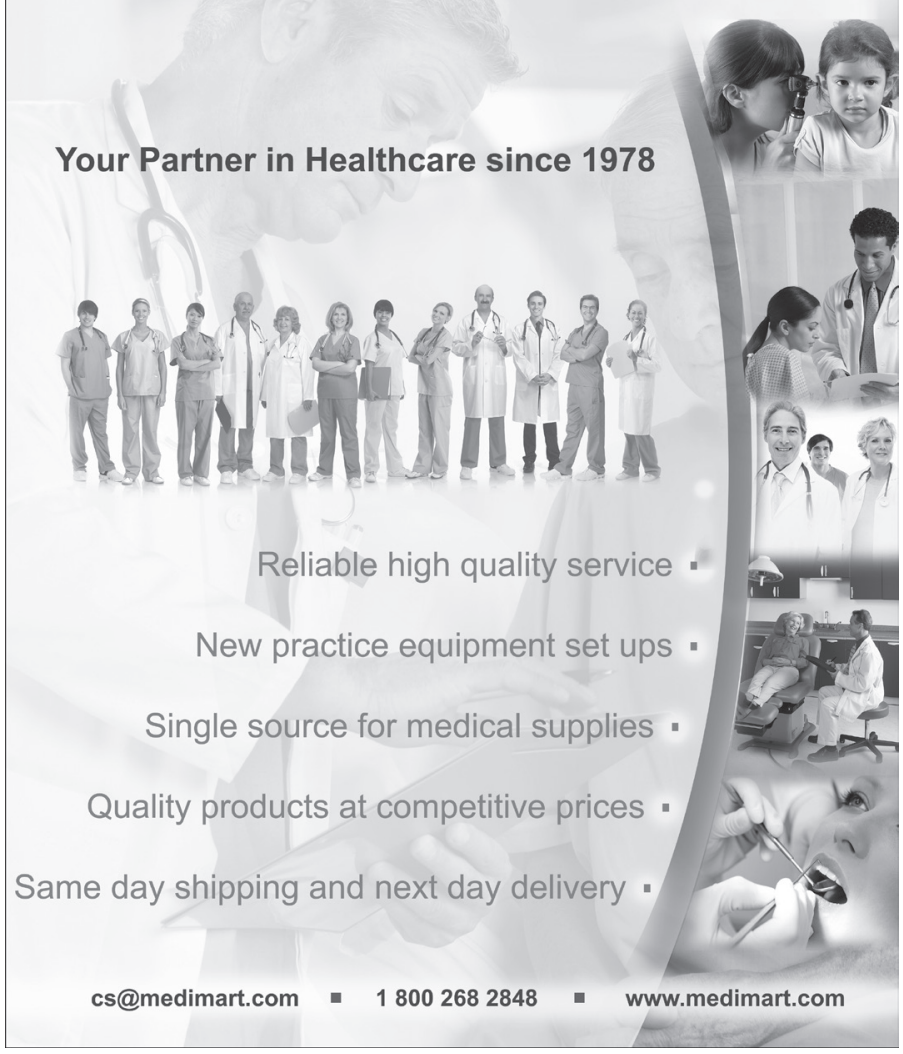

